\title{
Toxicidad en tratamientos combinados de abdomen y pelvis: riesgo/beneficio
}

\author{
A. Hervás, A. Montero, S. Sancho, R. Morera, J. A. Corona, S. Córdoba, I. Rodríguez, \\ E. Chajón, A. Ramos
}

\section{Introducción}

La cirugía junto con la radioterapia y la quimioterapia forman los tres pilares fundamentales en los que se basa la terapéutica del cáncer. En los tumores localmente avanzados los resultados a largo plazo son pobres. En un intento de mejorar la ganancia o índice terapéutico, es decir, el equilibrio entre toxicidad y control tumoral la radioterapia moderna se ha desarrollado en dos direcciones: por una parte lo que podríamos denominar avances tecnológicos propiamente dichos, como son los sistemas de inmovilización, la planificación tridimensional, la simulación virtual, la radioterapia conformada tridimensional, intensidad modulada de dosis (IMRT), los sistemas de control de calidad denominados de imagen portal, braquiterapia de alta tasa de dosis y pulsada, radiocirugía, radioterapia estereotáxica fraccionada, radiocirugía y radioterapia intraoperatoria y, por otro lado, nuevos avances terapéuticos: fraccionamientos alterados, radiosensibilizantes y radioprotectores, hipertermia, combinaciones con quimioterapia, fototerapia, partículas pesadas, terapias biológicas.

En los últimos 20 años y, hoy en pleno desarrollo, estamos asistiendo al uso de la utilización de la radioterapia y la quimioterapia de manera conjunta, como forma de mejorar la ganancia terapéutica. Los objetivos del tratamiento combinado son: mejorar el control locorregional, la preservación de órganos y su función, aumentar la supervivencia y disminuir las metástasis a distancia, todo ello intentando no aumentar la toxicidad con la combinación. Este tratamiento puede realizarse de manera neoadyuvante con la intención de actuar sobre el tumor primitivo disminuyendo su volumen y facilitando el efecto del tratamiento radioterápico y actuando a la vez sobre enfermedad metastásica o bien de forma concomitante mejorando la efectividad de la radioterapia sensibilizando su acción.

Servicio de Oncología Radioterápica

Hospital Ramón y Cajal

Madrid
Vamos a analizar la relación riesgo/beneficio del tratamiento radioquimioterápico en las localizaciones tumorales más frecuentes de abdomen y pelvis. Analizaremos la bibliografía más relevante y estableceremos unas recomendaciones basadas en los datos revisados.

\section{Cáncer de cérvix}

¿En las mujeres con cáncer de cérvix, en las cuales la radioterapia es el tratamiento de elección, la adición de quimioterapia concomitante basada en cisplatino mejora la supervivencia y la calidad de vida con toxicidad aceptable?

En Febrero de 1999 el NCl de EE.UU, basado en 5 ensayos controlados aleatorios estableció que en todas las pacientes con cáncer de cervix se debía tener en cuenta el uso de la radioquimioterapia concomitante.

En 2001, Green et al publicaron en Lancet una revisión sistemática y metaanálisis en la que se identificaron 19 ensayos (17 publicados y 2 no publicados) que incluían 4580 pacientes. La revisión sugiere firmemente que la radioquimioterapia mejora la supervivencia y la supervivencia libre de enfermedad con un beneficio absoluto del 12 y el $16 \%$ respectivamente. Hubo algunas pruebas de que el efecto fue mayor en los estudios que incluyeron una alta proporción de pacientes en estadios I y II. También redujo la recidiva local y a distancia. Se incrementa la toxicidad aguda pero los efectos secundarios a largo plazo fueron escasos.

Se identificaron 8 ensayos (uno incluía irradiación lumboaórtica y dos comparaban cisplatino frente a hidroxiurea además de radioterapia. Se analizaron un total de 2141 pacientes. Se encontraron diferencias en los estudios en cuanto a estadio de la enfermedad, régimen de quimioterapia (drogas y dosis), esquemas de radioterapia (dosis, tiempo total de tratamiento, braquiterapia), niveles de hemoglobina. El mataanálisis detectó una ventaja estadísticamente significativa en la supervivencia a favor del brazo con cisplatino (RR de muerte de 0.74, IC: 95\% (0.64-0.86). Reducción absoluta del riesgo de muerte: $11 \%$. También se objetivó en algunos estudios (Morris, Rose, Whitney, Peters) reducción en recidivas locales y a distancia. La toxicidad aguda G3-4 fue mayor en el brazo de tratamiento combinado, sobre todo hematológica y gastrointestinal. Solamente un estudio $(\mathrm{NCl}$ CTG) no observó beneficio del tratamiento combinado. No 
hay datos suficientes para apoyar el uso de 5-FU. En algunos estudios se utilizaron esquemas de radioterapia no óptimos. Los efectos del tratamiento en la calidad de vida sólo se evalúa en el NCIC CTG, pero los datos aún no han sido publicados. La toxicidad tardía: Tseng, $23 \%$ vs 13\% (proctitis, cistitis, fístula, obstrucción), Morris, $12 \%$ vs $11 \%$, Whitney, $16 \%$ ambos grupos de tratamiento.

Tras esta revisón podemos contestar afirmativamente a la pregunta planteada inicialmente, estableciendo las siguientes recomendaciones:

- A las pacientes con cáncer de cérvix que van a ser tratadas con radioterapia se les debe ofrecer tratamiento con cisplatino concurrente: pacientes con cáncer localmente avanzado, estadio bulky (>4 cm), estadios precoces con factores de alto riesgo (ganglios positivos o márgenes positivos) tras cirugía,.

- No existen comparaciones directas de distintos regímenes de cisplatino. Basados en la revisión de los datos de toxicidad disponible de ensayos aleatorizados se recomienda la utilización de cisplatino de forma semanal a dosis de 40 $\mathrm{mg} / \mathrm{m}^{2}$.

\section{Cáncer de recto}

¿En los pacientes con cáncer de recto la asociación de quimioterapia a la radioterapia mejora la supervivencia y la calidad de vida con toxicidad aceptable?

El tratamiento de elección del cáncer de recto es la cirugía. La supervivencia global a 5 años es del $85 \%, 60 \%$ y $40 \%$ para estadios I, II y III respectivamente. Las tasas de recidiva local a 5 años tras cirugía oscilan alrededor del 20$30 \%$ globalmente, siendo menores del $10 \%$ para $\mathrm{T} 1-2$ NO del $15-35 \%$ para T3N0 y del $45-65 \%$ para T3-4 N1-2. Por tanto, dado estos resultados, se plantea la necesidad de un tratamiento adyuvante.

Los resultados del NCCTG y del GITSG establecieron que para enfermedad localmente avanzada la radioquimioterapia postoperatoria consigue un aumento del control local y supervivencia comparado con cirugía sóla o cirugía más radioterapia. Tras estos resultados el NIH (National Institutes of Health) en 1990 en la "Conferencia de Consenso de tratamiento adyuvante para cáncer rectal de alto riesgo" recomendó el tratamiento con radioquimioterapia como estándar para pacientes con cáncer de recto, estadios II y III (Jama, 1990).

Posteriormente se iniciaron estudios con radioterapia preoperatoria. Las ventajas de esta opción terapéutica son las siguientes:

- biológicas: mejor oxigenación de las células tumorales.

- físicas: menos tóxica (menos adherencias y menor volumen de intestino irradiado).

- funcionales: posibilidad de conservación esfínter.

- datos europeos: menos costosa económicamente y más fácil de cumplimentar.

Las desventajas son:

- pérdida del estadio previo patológico.

- dificultad para indicación de quimioterapia adyuvante.

- sobretratamiento de pacientes.

- retraso del tratamiento más efectivo que es la cirugía.

Numerosos estudios aleatorizados han sido publicados con radioterapia preoperatoria objetivando una disminu- ción de la tasa de fracaso local cuando se compara con cirugía sóla. Sólo el estudio Sueco ha demostrado mejoría de la supervivencia (Swedish Rectal Cancer Trial, NEJM 1997). El DCCG ( Dutch Colorectal Cancer Group) objetivó que la combinación de radioterapia preoperatoria 5 ( 5 Gy en combinación con excisión mesorrectal completa (TME) redujo la tasa de fracaso local pero no mejoró la supervivencia. Recientemente se publicaron los resultados a 5 años de este estudio que confirma la disminución de la recidiva local pero no la mejora en la supervivencia (ESTRO 23).

Dadas las ventajas de la RT preoperatoria y los hallazgos de que la adición de quimioterapia a radioterapia mejora la supervivencia en situación postoperatoria se iniciaron estudios asociando radioquimioterapia en situación preoperatoria. De éstos el más importante es el publicado por el German Rectal Cancer Study Group en que comparan RT/QT preoperatoria (50.4 Gy + 5-FU) versus RT/QT postoperatoria (55.8 Gy + 5-FU). Se analizan 421 pacientes. No hubo diferencias en la SG a 5 años (76\% vs $74 \%$ ). Sí la hubo a favor del tratamiento preoperatorio en la tasa de recidiva locorregional a 5 años ( $6 \%$ vs 13\%, p=0.006), en la toxicidad aguda G3-4 (27\% vs $40 \%, \mathrm{p}=0.001)$, toxicidad tardía $(14 \%$ vs $24 \%, p=0.01)$, cumplimiento del tratamiento RT (92\% vs $54 \%, \mathrm{p}<0.001)$ y cumplimiento del tratamiento QT $(89 \%$ vs $50 \%$ p<0.001) (Sauer R et al, NEJM 2004), la preservación de esfínter ( $39 \%$ vs $19 \% \mathrm{p}=0.04)$.

Por tanto, tras estos datos, podemos concluir que:

- A los pacientes con cáncer de recto estadios II y III se les debe ofrecer tratamiento con radioquimioterapia preoperatoria porque aunque no ha demostrado beneficio en la supervivencia ni mejoría de las recidivas sistémicas presenta otras ventajas:

- Mejor cumplimiento del tratamiento

- Disminución del estadio: preservación de esfínter

- Disminución de la toxicidad

- Mayor control local

Se recomiendan dosis de 45-50.4 Gy y 5-FU en IC como tratamiento estándar, fuera de ensayo clínico.

\section{Cáncer de páncreas}

En este apartado vamos a analizar por separado el cáncer de páncreas irresecable y el resecable.

¿En los pacientes con cáncer de páncreas localmente avanzado (irresecables) la asociación de quimioterapia y radioterapia mejora la supervivencia y la calidad de vida con toxicidad aceptable?

Existen pocos estudios y de mala calidad. En la "Practice Guidelines" publicada por Cancer Care Ontario Program en 2004 analizan 14 ensayos aleatorizados. La conclusión de esta revisión es que:

- la RT/QT es mejor que RT o QT sóla.

- RT/QT con 5-FU no fue superada por otro esquemas.

- QT vs poliQT: más toxicidad y ningún beneficio.

- Se recomienda el 5-FU como sensibilizante fuera de ensayo clínico.

Críticas a esos estudios:

- Ningún estudio compara QT vs RT 


\section{A. Hervás y cols.}

- Los ensayos aleatorizados con QT exclusiva incluyen pacientes con cáncer metastásico y localmente avanzado y no los analiza separadamente.

- Pocos estudios y con poca potencia estadística para obtener conclusiones.

- Imposibilidad de metaanálisis por series muy heterogéneas desde el punto de vista clínico y terapéutico.

- Son estudios muy antiguos: año 69 y década de los 80 .

- Las técnicas de RT y prescripción de dosis son absolutamente inadecuadas para los estándares actuales: Co-60 en la mayoría de los casos, campos AP-PA, no planificación 3D y ningún control de calidad del tratamiento RT.

- Pocos incluyen estudios de calidad de vida.

- Ninguno valora tasa de resecabilidad tras el tratamiento.

Respecto a la pregunta inicial, tras estos datos, podríamos hacer las siguientes recomendaciones:

- En pacientes sintomáticos y con mal estado general: QT vs tratamiento de soporte.

- Pacientes con buen P.S.: RT/QT con 5-FU en IC o en bolus, ya que algunos estudios han demostrado mejoría de la supervivencia aunque modesta.

- No se debe recomendar QT o RT sóla, aunque la QT sóla con Gemcitabina puede ser una alternativa aceptable.

¿En los pacientes con cáncer de páncreas resecados la asociación de quimioterapia y radioterapia postoperatoria mejora la supervivencia y la calidad de vida con toxicidad aceptable?

En el año 1985 se publicó un ensayo aleatorizado del GITSG en el que incluyeron 49 pacientes donde comparaban RTE (40 Gy en split course) y QT (5-FU en bolo 500 $\mathrm{mg} / \mathrm{m}^{2}$ los 3 primeros días de cada curso de RTE y durante 2 años de mantenimiento vs observación. Los resultados mostraron un beneficio en la supervivencia a 2 años a favor del grupo de tratamiento $(42 \%$ vs $15 \%, \mathrm{p}=0.03)$. Las críticas a ese estudio son: defectos metodológicos, bajas dosis de RT, escaso número de pacientes incluídos, periodo de inclusión largo.

La EORTC en su estudio 40891 intentó reproducir los resultados del GITSG. Se incluyeron 218 pacientes con el mismo esquema de tratamiento, aunque no se utilizó la QT de mantenimiento. Este estudio no demostró diferencias significativas en la supervivencia.

En 2001 se publicaron los resultados del controvertido ensayo ESPAC-1 (Neptolemos, Lancet 2001). Se trataba de tres estudios paralelos tras la resección quirúrgica. El primer estudio con 68 pacientes comparaba RT/QT con RTE (40 Gy con split course) y 5 -FU vs observación (esquema del GITSG). El segundo estudio con 168 pacientes comparaba QT adyuvante con 5 -FU vs observación y el tercer estudio con 288 pacientes era un diseño multifactorial 2 ( 2 en el que se comparaban RT/QT, QT, ambos y observación. De los resultados preliminares publicados, con un seguimiento mediano de 10 meses, es difícil extraer conclusiones. Parece que RT/QT no es satisfactoria tras cirugía como tratamiento adyuvante. Las críticas a ese estudio fueron:

- El médico seleccionaba el grupo de inclusión del paciente.

- Elegía según experiencia: QT/RT oQT

- La RT se administraba en split-course.
- Dosis de 40-60 Gy a criterio del especialista.

- El grupo de RT/QT no incluyó QT adyuvante (no puede compararse con GITSG).

En 2004 se publicaron los resultados finales de este estudio, con un seguimiento mediano de 47 meses, con un beneficio en la supervivencia a 5 años a favor del brazo que no recibió RT/QT (10\% vs $20 \%$, $\mathrm{p}=0.05$ ) y a favor del brazo de tratamiento con quimioterapia ( $21 \%$ vs $8 \%, p=0.009)$. Las conclusiones de este estudio son que la QT adyuvante mejora significativamente la supervivencia en pacientes con cáncer de páncreas resecado y que la radioquimioterapia tiene un efecto perjudicial sobre la supervivencia en estos pacientes.

La RTOG ha cerrado un ensayo aleatorizado, el 97-04, activado en Agosto de 1988 cuyo objetivo es establecer el mejor esquema de RT/QT adyuvante tras resección quirúrgica, se comparan dos esquemas de radioquimioterapia RT/QT 50.4 Gy + 5-FU en IC en ambos brazos y Gemcitabina antes y después de RT/QT por 3 meses frente al mismo esquema de RT/QT con 5-FU antes y 3 meses después de RT/QT. Se han comunicado sólo datos de toxicidad, siendo más alta en el brazo de Gemcitabina.

Por tanto, tras este análisis no podemos recomendar como tratamiento estándar la radioquimioterapia en pacientes con cáncer de páncreas resecados, aunque en EE.UU. es el tratamiento de elección basados en los resultados del GITSG. Actualmente no hay publicados ensayos aleatorizados recientes que confirmen esos datos. Los resultados finales del ESPAC 1 hay que tomarlos con cautela dada la presencia de grandes sesgos: fallos en la aleatorización, alta tasa de no adherencia al tratamiento, cambios de tratamiento en los grupos, técnica y dosis de RT obsoletas.

\section{Cáncer gástrico}

¿En los pacientes con cáncer gástrico la asociación de quimioterapia y radioterapia mejora la supervivencia y la calidad de vida con toxicidad aceptable?

En pacientes con cáncer gástrico resecable la cirugía es el tratamiento de elección. Actualmente existe controversia sobre el tipo de linfadenectomía más adecuado. Tiene valor pronóstico y nos ayuda en la precisión del estadio. En base a los trabajos publicados es difícil justificar una linfadenectomía extendida; sin embargo, puede asumirse que una linfadenectomía con no menos de 15 ganglios podría ser adecuada. El 60\% de los pacientes $\mathrm{N}+$ y/o T3-4 recidivan en el lecho quirúrgico, ganglios regionales, muñón o anastomosis. Esto justifica la necesidad de un tratamiento adyuvante.

Existen estudios aleatorizados con radioterapia adyuvante tras cirugía (Hallissey, Kramling, Bleiberg). A pesar de sus defectos metodológicos se puede concluir que la radioterapia aumenta el control local aunque sin modificar la supervivencia, la importancia de administrar dosis suficientes de la misma y el papel decisivo de la cirugía radical en los resultados finales.

También han sido publicados numerosos ensayos comparando quimioterapia sistémica vs observación en pacientes con cáncer gástrico resecado. Existen 4 metaanálisis (Herman, Earle, Mari y Gianni) en los que se objetiva un beneficio marginal en la supervivencia, siendo mayor este beneficio en los pacientes con ganglios positivos. Los efectos adver- 
sos pueden ser significativos, recibiendo menos del $80 \%$ las dosis previstas.

Respecto al tratamiento adyuvante con radioquimioterapia han sido publicados algunos ensayos aleatorizados (Dent, Moertel, Hallissey) en los que se refiere alto incumplimiento del esquema por toxicidad y no se objetivan diferencias en la supervivencia.

El estudio aleatorizado más reciente con radioquimioterapia es el del INT 0116 publicado en 2001 (Mc Donald, NEJM). Es un estudio fase III con 556 pacientes T3-4, N1-2 en el que los pacientes se aleatorizan a RT/QT postoperatoria (45 Gy + 5-FU) u observación. Los resultados a 3 años mostraron mejor SG, SLE, supervivencia media y supervivencia media libre de progresión a favor del brazo de tratamiento. No hubo diferencias en las recidivas locorregionales ni a distancia. Las críticas a este estudio son las siguientes:

- La ventaja terapéutica de la rama del tratamiento combinado puede estar detectando el efecto terapéutico de la RT/QT postoperatoria sobre una cirugía insuficiente (el 54\% de los pacientes tienen linfadenectomía inferior a D1).

- Elevada toxicidad: sólo el 64\% completaron el tratamiento, G3 (41\%) y G4 (32\%), G3-G4 (73\%).

- La quimioterapia utilizada (5-FU/LV bolus) no es activa en enfermedad avanzada ni es la más adecuada para combinar con radioterapia.

Park et al en 2003 publicaron un estudio reproduciendo el esquema del INT 0116 . Es un estudio prospectivo, no aleatorizado con 290 pacientes. Con un seguimiento medio de 49 meses el $80 \%$ completan el tratamiento. Toxicidad hematológica G3 (30\%) y digestiva G3 (38\%). La SG y SLE a 5 años fue del 60 y $57 \%$ respectivamente. Las recidivas locorregionales fueron del $29 \%$, peritoneales $67 \%$ y a distancia $36 \%$. Recientemente se ha publicado otro estudio prospectivo con el mismo propósito objetivando que con la radioterapia conformada se mejora la toxicidad aguda, siendo los datos de supervivencia superponibles a los del INT-0116.

Respecto al tratamiento neoadyuvante con radioquimioterapia actualmente sólo existen datos de ensayos no aleatorios que sugieren la posibilidad de un beneficio en la supervivencia con tratamiento combinado para pacientes con cáncer gástrico localizado.

En respuesta a la pregunta inicial podríamos establecer las siguientes conclusiones:

- Los resultados del INT 0116 , que demostró un beneficio en la supervivencia, establecieron el tratamiento estándar en EEUU para pacientes diagnosticados de cáncer gástrico que han sido sometidos a una cirugía potencialmente curativa.

- Aunque el régimen más adecuado no ha sido definido, en los actuales protocolos se recomienda IC de 5-FU durante la irradiación externa $\left(200-225 \mathrm{mg} / \mathrm{m}^{2}\right)$.

- Para pacientes no candidatos a RT/QT la QT adyuvante podría ser beneficiosa aunque el régimen óptimo permenece por definir.

- La QT adyuvante postoperatoria es el tratamiento de elección en Japón y algunas partes de Europa.

- Los datos disponibles sugieren que el beneficio de la QT puede ser mayor en pacientes con metástasis ganglionares.

- No hay evidencia suficiente actualmente de ensayos aleatorizados para recomendar el tratamiento neoadyuvante fuera de ensayos clínicos. Los resultados de los ensayos en marcha ayudarán a definir mejor su papel.
Como conclusiones generales sobre el tratamiento combinado de radioquimioterapia en abdomen y pelvis podemos establecer que:

- Los tratamientos combinados en abdomen y pelvis, en general, producen aumento del índice terapéutico. Actualmente esa ganancia terapéutica llegará a ser mayor derivada de las mejoras a nivel tecnológico y farmacológico (nuevas drogas y terapias de soporte).

- El tratamiento combinado debe ofrecerse a pacientes con buen PS y a los cuales se les pueda facilitar un adecuado tratamiento de soporte y estrecho seguimiento.

- No debemos olvidarnos de las toxicidades crónicas que rara vez son reflejadas en los estudios y que pueden afectar en gran medida a la calidad de vida de los pacientes.

- Las investigaciones futuras deberán ir dirigidas a la integración adecuada de las distintas modalidades de tratamiento (cirugía, radioterapia y quimioterapia) y optimización de las mismas, así como al desarrollo de tratamientos derivados de los avances logrados en biología molecular.

\section{Bibliografía}

1. Steel GG, Peckham MJ. Explotaible mechanism in combined radiotherapy-chemotherapy. The concep of additivity. Int J Radiat Oncol Biol Phys 1979; 5:85-91.

2. Tannock IF. Treatment of cancer with radiation and drugs. J Clin Oncol 1996; 14:3156-3174.

3. Martin Brown J. Therapeutic targets in radiotherapy. Int J Radiat Oncol Biol Phys 2001; 49: 319-326.

4. Morris M, Eifel PJ, LU J et al. Pelvic radiation with concurent chemotherapy compared with pelvic and para-aortic radiation for high-risk cervical cancer. N Engl J Med 1999; 340: 113742

5. Rose PG, Bundy BN, Watkins EB et al. Concurrent cisplatin-based radiotherapy and chemotherapy for locally advanced cervical cancer. N Engl J Med 1999: 340: 1144-53.

6. Whitney CW, Sause W, Bundy BN et al. Randomized comparison of fluorouracil plus cisplatin versus hydroxiurea as an adjunct to radiation therapy in stage IIB-IV A carcinoma of the cervix with negative para-aortic lymph nodes: a Gynecologic Oncoloic Group and Southwest Oncology Group study. J Clin Oncol 1999: 17; 1339-48.

7. Keys HM, Bundy BN, Stehman FB et al. Cisplatin, radiation and adjuvant hysterectomy for bulky stage IB cervical carcinoma. $\mathrm{N}$ Engl J Med 1999; 340: 1154-61.

8. Peters WA, Liu PY, Barret R et al. Concurrent chemotherapy and pelvic radiation therapy compared with pelvic radiation therapy alone as adjuvant therapy after radical surgery in highrisk early-stage cancer of the cervix. J Clin Oncol 2000; 18: 1606-13.

9. Pearcey $R$, Brundage $M$, Drouin P et al. A clinical trial comparing concurrent cisplatin and radiation therapy versus radiation alone for locally advanced squamous cell carcinoma of the cervix carried out by the National Cancer Institute of canada Trials Group (abstract). Proc Am Soc Clin Oncol 2000: 19; 378a. Abstract 1497

10. Wong LC, Choo YC, Choy D et al. Long-term follow-up of potentiation of radiotherapy by cis-platinum in advanced cervical cancer. Gynecol Oncol 1989; 35: 159-63. 
11. Tseng $\mathrm{C}-\mathrm{H}$, Chang $\mathrm{C}-\mathrm{T}$, Lai $\mathrm{C}-\mathrm{H}$ et al. A randomized trial of concurrent chemoradiotherapy versus radiotherapy in advanced carcinoma of the uterine cervix. Gynecol Oncol 1997; 66:52-8.

12. Gren JA, Kirwan JM, Tierney JF et al. Survival and recurrence after concomitant chemotherapy and radiotherapy for cancer of the uterine cervix: a systematic review and meta-analysis. Lancet, 2001; 358: 781-786.

13. H Lukka, H Hirte, A Fyles et al. Primary treatment for locally advanced cervical cancer: concurrent platinum-based chemotherapy and radiation: Practice Guideline Report \#4-5. A cancer care ontario program.

14. Gastrointestinal Tumor Study Group. Prolongation of the disease-free interval in surgically treated rectal carcinoma. N Engl J Med 1985; 312: 1465-72.

15. Krook JE, Moertel CG, Gunderson LL et al. Effective surgical adjuvant therapy for high-risk rectal carcinoma. $N$ Engl J Med 1991; 324: 709-15.

16. NIH consensus conference: adjuvant therapy for patients with colon and rectal cancer. JAMA 1990; 264:1444-50.

17. Swedish Rectal Cancer Trial. Improved survival with preoperative radiotherapy in resectable rectal cancer. $N$ Engl J Med 1997; 336: 980-7.

18. Colorectal Cancer Collaborative Group. Adjuvant radiotherapy for rectal cancer: a systematic overview of 8507 patients from 22 radndomized trials. Lancet 2001; 358:1291304

19. Marijnen CAM, Peeters KCMJ, Putter $\mathrm{H}$ et al. Long term results, toxicity and quality of life in the TME trial. Radiother and Oncol 2004; 73 (supplement 1) S127

20. Saver R, Becker H, Hohenberger $W$ et al. Preoperative versus Postoperative Chemoradiotherapy for Rectal Cancer. N Engl J Med 2004; 351: 17311740.

21. Earle CC, Agboola O, Maroun J et al. The treatment of locally advanced pancreatic cancer. Practice Guideline Report\#2-7. Cancer Care Ontario Program.

22. Kalser MH, Ellenberg SS. Pancreatic cancer: adjuvant combi- ned radiation and chemotherapy following curative resection. Arch Surg 1985; 120: 899-903.

23. Yeo CJ, Abrams RA, Grochow LB et al. Pancreaticoduodenectomy for pancreatic adenocarcinoma: postoperative adjuvant chemoradiation improves survival: a prospective, single-institution experience. Ann Surg 1997; 225: 621-33.

24. Klimkenbiil JH, Jeekel J, Sahmoud T et al. Adjuvant radiotherapy and 5-fluorouracilafter curative resection of cancer of the pancreas and periampullary region: phase III trial of the EORTC Gastrointestinal Tract Cancer Cooperative Group. Ann Surg 1999; 230: 776-82.

25. Neoptolemos JP, Dunn JA, Stocken DD, et al. Adjuvant chemoradiotherapy and chemotherapy in resectable pancreatic cancer: a randomised controlled trial. Lancet 2001; 358:1576-85.

26. Neoptolemos JP, Stocken DD, Friess $\mathrm{H}$, et al. A randomised trial of chemoradiotherapy and chemotherapy after resection of pancreatic cancer. N Engl J Med 2004; 350:1200-10.

27. Choti M. Adjuvant therapy for pancreatic cancer- The debate continues. N Engl J Med 2004; 350: 1249-1251.

28. The Gastrointestinal Tumor Study Group. Controlled trial of adjuvant chemotherapy following curative resection for gastric cancer. Cancer 1982; 49: 1116-1122.

29. Gunderson L, Sosin H. Adenocarcinoma of the stomach: areas of failure in a re-operation series. Int J Rasiat Oncol Biol Phys 1982;8:1-11.

30. Mac Donald J et al. Postoperative combined radiation and chemotherapy improves disease-free survival (DFS) and overall survival (OS) in resected adenocarcinoma of the stomach and GE junction. N Engl J Med 2001; 345:725-30.

31. Park SH, Kim DY, Heo JS et al. Postoperative chemotradiotherapy for gastric cancer. Ann Oncol 2003: 14:1373-7.

32. Earle $C$ et al. Adjuvant chemotherapy after curative resection for gastric cancer: Revisiting a meta-analysis of randomized trials. Proc ASCO 1998; 17:263a.

33. Ringash J, Khaksar SJ, Oza A, et al. Post-operative Radiochemotherapy for Gastric Cancer: Adoption and Adaption. Clin Oncol 2005; 17:91-95. 